\title{
Securin promotes migration and invasion via matrix metalloproteinases in glioma cells
}

\author{
HAICHENG YAN $^{1 *}$, WEI WANG ${ }^{2 *}$, CHANGWU DOU $^{3}$, FUMING TIAN $^{3}$ and SONGTAO QI ${ }^{1}$ \\ ${ }^{1}$ Department of Neurosurgery, Nanfang Hospital, Southern Medical University, Guangzhou, Guangdong 510515; \\ ${ }^{2}$ Department of Oncology, Inner Mongolia People's Hospital, Hohhot, Inner Mongolia 010017; \\ ${ }^{3}$ Department of Neurosurgery, Affiliated Hospital of Inner Mongolia Medical University, \\ Hohhot, Inner Mongolia 010051, P.R. China
}

Received June 26, 2014; Accepted March 10, 2015

DOI: $10.3892 / 01.2015 .3074$

\begin{abstract}
Human securin, encoded by pituitary tumor transforming gene 1 , is implicated in several oncogenic processes in the pathogenesis of brain tumors, including glioma. The aim of the present study was to examine the effect of securin on the migration and invasion of glioma cells. The results revealed that the overexpression of securin in glioma LN-229 cells significantly increased the invasion and transmigration abilities. By contrast, these abilities were significantly reduced by the downregulation of securin in glioma U373 cells. Furthermore, the results demonstrated that securin overexpression and downregulation significantly increased and decreased the levels of matrix metalloproteinase 2 and 9, respectively. These findings indicate a promotive role for securin in glioma migration and invasion, which may involve the action of matrix metalloproteinases.
\end{abstract}

\section{Introduction}

Pituitary tumor transforming gene 1 (PTTG1) is the human homolog of securin and was originally identified in rat pituitary adenoma cells (1). PTTG1/securin is highly expressed in a variety of human malignancies, including those of the breast (2), uterus (3), lung (4), thyroid (5) and brain (6). A high expression level of securin has been revealed to be correlated with extremely poor outcomes in breast cancer, adrenocortical cancer $(7,8)$ and glioma (6) patients. A previous study demonstrated that the transgenic overexpression of PTTG1 in the mouse pituitary caused early multipotential pituitary

Correspondence to: Dr Songtao Qi, Department of Neurosurgery, Nanfang Hospital, Southern Medical University, 1,838 North Guangzhou Avenue, Guangzhou, Guangdong 510515, P.R. China

E-mail: qi_songtao@126.com

*Contributed equally

Key words: glioma, matrix metalloproteinase, pituitary tumor transforming gene 1 , securin focal hyperplasia (9). By contrast, it was also previously identified that the downregulation of securin reduced angiogenic activity in pluripotent stem cell-derived vascular endothelial cells (10). In addition, a further study established that PTTG1 deficiency protected against pituitary tumorigenesis induced by retinoblastoma haploinsufficiency (11). In the glioma U87 cell line, PTTG-knockdown had an inhibitive effect on serum-induced proliferation, and PTTG expression was upregulated by the promalignant ligands, epithelial growth factor and transforming growth factor- $\alpha$ (12). These results indicate a promotive role for PTTG1/securin in oncogenic processes.

Invasion is an important characteristic of malignant tumors, which involves the proteolytic degradation of extracellular matrix (ECM) components. Matrix metalloproteinases (MMPs) are regulators of ECM proteins. MMPs are members of a large family of proteolytic enzymes that degrade the majority of ECM components. At present, six groups of MMPs have been identified; these include collagenases, matrilysins, stromelysins, gelatinases, membrane-type matrix metalloproteinases, and other MMPs. MMP2 (also known as gelatinase A) and MMP9 (also known as gelatinase B) cleave denatured collagens (gelatins), laminins and certain chemokines (13). In addition, MMP2 activates MMP1 and MMP9 by cleaving their prodomains (14). Under physiological conditions, MMP expression is low in the majority of normal cells. By contrast, MMP expression is markedly increased in a number of different cancers $(15,16)$ and is directly associated with tumor invasiveness (17), which contributes to poor patient outcomes. As two important members of the MMP family, MMP2 and MMP9 have been identified to be involved in colon cancer progression, hepatic metastasis (18), the promotion of lung cancer invasion (19), and hepatocellular carcinoma cell migration and invasion (20). MMP2 and 9 have also been revealed to participate in invasion. The findings of a previous study established that MMP2 exhibited a positive correlation with $33 \mathrm{GnT} 8$ in glioma U251 cells, and that silencing of the latter decreased cell proliferation, migration and metastatic ability (21). A further study identified that the cytotoxic treatment of U251 cells significantly reduced MMP2 and MMP9 expression (22).

The association between securin and MMPs has previously been reported. Certain studies identified that PTTG, MMP2 
and MMP9 were simultaneously overexpressed in metastatic papillary thyroid carcinomas $(23,24)$. In HEK293 cells, transfection with PTTG cDNA significantly increased the secretion and expression of MMP2 (25). Furthermore, in a cutaneous squamous cell carcinoma cell line, the downregulation of PTTG by small interfering RNA (siRNA) significantly inhibited proliferation and metastasis, and decreased the expression of MMP2 and MMP9 (26). These findings indicate a positive correlation between securin and MMPs. In order to further understand the role of securin in glioma, the present study established models of securin overexpression and downregulation in different glioma cells using securin cDNA and siRNA, respectively. Using these models, the effect of securin on glioma migration and invasion, and the potential role of MMPs, was investigated.

\section{Materials and methods}

Reagents. The chemiluminescence reagent was purchased from Pierce Biotechnology, Inc. (Rockford, IL, USA) and G418 was purchased from Sigma-Aldrich (St. Louis, MO, USA). The fetal bovine serum (FBS), RPMI-1640 medium, Lipofectamine 2000, TRIzol reagent and SYBR GreenER qPCR SuperMix Universal kit (cat. no. 11762-500) were purchased from Life Technologies (Grand Island, NY, USA). M-MLV reverse transcriptase was purchased from Promega Corporation (Madison, WI, USA). The normal human astrocyte (NHA) cells and glioma LN-229, U87, U251 and U373 cell lines were purchased from the American Type Culture Collection (Manassas, VA, USA). The pGenesil 2 and pcDNA3.1 plasmids were purchased from Jingsai Shenggong Biological Co., Ltd. (Shanghai, China). Polyclonal primary rabbit anti-human antibodies including anti-AKT (product no. 4691), anti-phosphorylated (phospho) AKT (Ser473; product no. 4060), anti- $\beta$ actin (product no. 4967), antiMMP2 (product no. 4022), anti-MMP 9 (product no. 3852) or anti-securin (product no. 13445) were purchased from Cell Signaling Technology, Inc. (Danvers, MA, USA), while the peroxidase conjugated rabbit IgG was purchased from Santa Cruz Biotechnology, Inc. (Dallas, Texas, USA).

Quantitative polymerase chain reaction ( $q P C R)$. The mRNA levels were investigated using qPCR, as previously described (13). The total RNA was extracted using TRIzol reagent according to the manufacturer's instructions. The RNA was first reverse transcribed into cDNA using M-MLV reverse transcriptase. Next, qPCR was performed using the SYBR GreenER qPCR SuperMix Universal kit with an ABI StepOnePlus real-time PCR system (Applied Biosystems Life Technologies, Foster City, CA, USA). Subsequent to an initial denaturation at $95^{\circ} \mathrm{C}$ for $5 \mathrm{~min}$, the following cycling profile was used: Denaturation at $95^{\circ} \mathrm{C}$ for $30 \mathrm{sec}$, annealing at $60^{\circ} \mathrm{C}$ for $30 \mathrm{sec}$ and extension at $72^{\circ} \mathrm{C}$ for $45 \mathrm{sec}$. Amplification was performed for 39 cycles. The primers specific for the target genes are shown in Table I. The results are presented as the levels of expression relative to those of the controls, subsequent to normalization to $\beta$-actin using the $2^{-\Delta \Delta \mathrm{Ct}}$ method.

Western blot analysis. The protein levels of phospho-AKT, MMP2, MMP9 and securin were analyzed using western blotting, as previously described (13). Whole tissue or cell proteins were electrophoretically separated using 4-12\% SDS-PAGE gels and then transferred to nitrocellulose membranes. Subsequent to 30 min of blocking with $2.5 \%$ skimmed milk, the membranes were incubated with the appropriate primary antibody (dilution, 1:2,000) overnight at $4{ }^{\circ} \mathrm{C}$, followed by a 1-h incubation with a horseradish peroxidase-conjugated secondary antibody (dilution, 1:2,000). Subsequent to treatment with each antibody, the membranes were washed with phosphate-buffered saline (PBS) containing 0.5\% Tween 20. Next, the membranes were developed using a chemiluminescence reagent and then exposed to X-ray film. The protein levels are expressed as the ratio of the band optical intensity to that of $\beta$-actin or total AKT.

Stable securin transfection in LN-229 cells. Complete securin cDNA was cloned into pcDNA3.1 plasmids and confirmed by DNA sequencing. The stably transfected LN-229 cells were selected using neomycin. Briefly, the LN-229 cells were transfected with a securin-pcDNA3.1 plasmid or an empty pcDNA3.1 vector using Lipofectamine 2000 according to the manufacturer's instructions. Subsequent to $24 \mathrm{~h}$ of transfection, the cells were trypsinized and reseeded into a 6-well plate. These cells were selected using a selection medium containing $600 \mu \mathrm{g} / \mathrm{ml} \mathrm{G} 418$. The surviving cell clones, which were considered to be the stably transfected cells, were maintained and passaged in a culture medium containing $300 \mu \mathrm{g} / \mathrm{ml} \mathrm{G} 418$.

Transfection of siRNA in U373 cells. siRNA sequences specific to human securin were designed according to the following securin target sequences: Sequence \#1, TGGGAGATCTCA AGTTTCA; sequence \#2, GTCTGTAAAGACCAAGGGA; and sequence \#3, GCATTCTGTCGACCCTGGA. These siRNA oligonucleotides were cloned into pGenesil 2 plasmids. In a 6 -well plate, $2 \times 10^{5} \mathrm{U} 373$ cells were seeded to $50-60 \%$ confluence in antibiotic-free RPMI-1640 medium and left to grow for $24 \mathrm{~h}$. The cells in each well were then transfected with 200 pmol securin siRNA or scramble siRNA using Lipofectamine 2000, according to the manufacturer's instructions. The medium was changed $6 \mathrm{~h}$ after transfection and the cells were incubated for an additional $48 \mathrm{~h}$ until further use.

Migration assay. Cell migration was assessed using a wound-healing scratch assay. The LN-229 cells with stable securin transfection and the U373 cells with securin siRNA transient transfection were plated into 6-well plates and allowed to grow until $90-95 \%$ confluence was reached. The cells were then washed in PBS. Next, the medium was replaced with serum-free medium and the cells were grown for an additional $24 \mathrm{~h}$. Scratches were then made using a P200 pipette tip (Rainin, Oakland, CA, USA). The images were captured at the 0-, 18- and 36-h time-points with a microcamera (Olympus, Waltham, Massachusetts).

Invasion assay. Cell invasion was assessed using the Transwell invasion assay. The LN-229 and U373 cells were trypsinized and resuspended in FBS-free RPMI-1640 medium $48 \mathrm{~h}$ after transfection. In total, $2 \times 10^{4}$ cells were plated into the upper chamber of the Transwell unit, which contained a Matrigel-coated polycarbonate membrane. Next, fresh medium containing $10 \%$ FBS was added to the lower chamber 
Table I. Primer sequences.

Sense $\left(5^{\prime} \rightarrow 3^{\prime}\right)$

Antisense $\left(5^{\prime} \rightarrow 3^{\prime}\right)$

Accession number

\begin{tabular}{lllc}
\hline$\beta$-actin & GCTCTTTTCCAGCCTTCCTT & GAGCCAGAGCAGTGATCTCC & HQ154074 \\
MMP2 & CTCCACTGGATGGAGGAAAA & ACGGCCACTCAGTAGGTGTC & NM004530 \\
MMP9 & GAGTTCCCGGAGTGAGTTGA & ACTCCTCCCTTTCCTCCAGA & NM004994 \\
Securin & AAAGCTCTGTTCCTGCCTCA & GAGAGGCACTCCACTCAAGG & NM001282383 \\
\hline
\end{tabular}

MMP, matrix metalloproteinase.

A
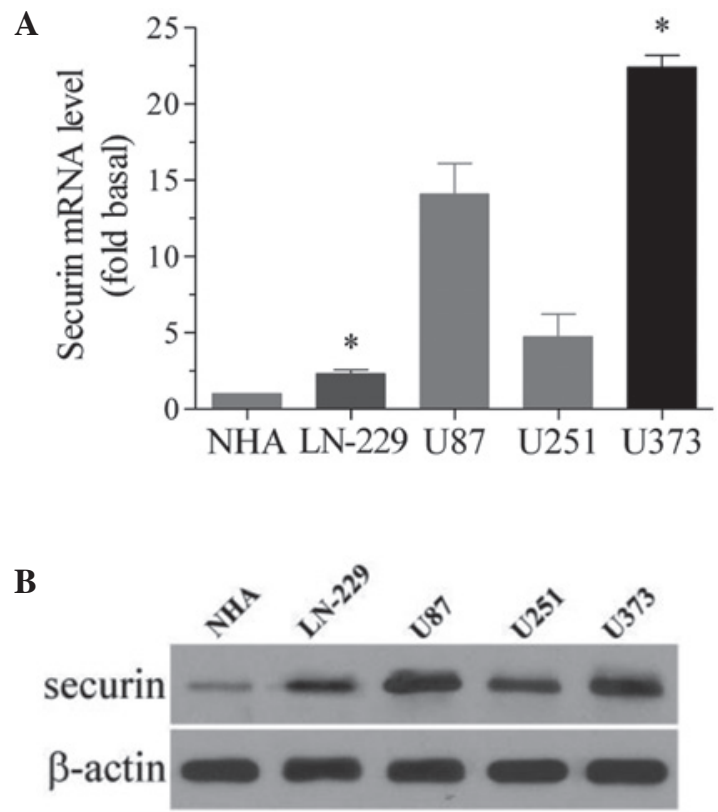

C

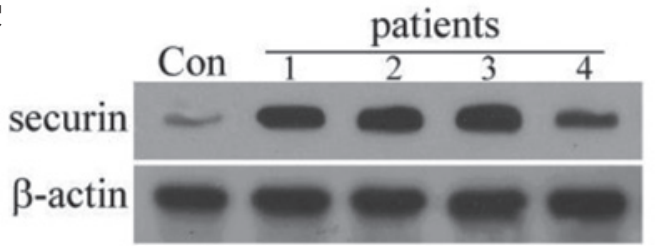

Figure 1. Expression of securin in glioma cells and tissues. (A) mRNA and (B) protein expression of securin in different glioma cells was investigated using quantitative polymerase chain reaction and western blot analysis, respectively $(n=4)$. (C) The protein expression of securin in glioma tissues obtained from patients $(n=40)$ was assessed using western blot analysis. Tissues from brain trauma patients were used as the control $(n=10)$. Representative immunoblots are shown. $\mathrm{P}<0.05$ vs. control group. ${ }^{*} \mathrm{P}<0.05$ vs. LN-229, U87 and U251 groups. NHA, normal human astrocyte; Con, control.

to act as a chemoattractant. Subsequent to a 48-h incubation at $37^{\circ} \mathrm{C}$ with $5 \% \mathrm{CO}_{2}$, the cells on the lower surface of the membrane were fixed with $5 \%$ formalin and stained with $0.2 \%$ crystal violet. The non-migrated cells on the upper surface of the membrane were removed with a cotton swab. Images of the cells that had migrated to the lower surface were captured with a microcamera (Olympus). The number of invading cells was counted from six randomly-selected fields.

Ethics statement. The glioma tissues and normal brain tissues used in the present study were obtained from glioma and brain trauma patients, respectively. These patients agreed with the study, and signed informed consent forms. The study protocol was approved by the Institutional Review Board of Nanfang Hospital (Guangzhou, China).

Statistical analysis. Graphpad Prism software version 5 was used for the data analysis. The data are presented as the mean \pm standard error of the mean. The statistical analysis was performed using a one-way analysis of variance, followed by Dunnett's test for multiple comparisons and Student's t-test for comparisons between groups. $\mathrm{P}<0.05$ was used to indicate a statistically significant difference.

\section{Results}

Securin expression in various glioma cells and human brain glioma tissues. The expression of securin was analyzed in a number of glioma cells. qPCR revealed that the expression of securin mRNA was significantly higher in the four types of glioma cells (LN-229, U87, U251 and U373) compared with the NHA cells $(\mathrm{P}<0.05$; Fig. 1A). Of the four types of glioma cells, LN-229 cells exhibited the lowest expression of securin, whereas U373 cells demonstrated the highest expression of securin. Therefore, LN-229 and U373 cells were used for the subsequent analysis of securin overexpression and downregulation. In the western blot analysis, the protein expression of securin was also higher in the four types of glioma cells than in the NHA cells (Fig. 1B). The present study also used western blot analysis to investigate whether an increased expression level of securin was evident in human glioma tissues. Compared with the brain tissues obtained from brain trauma patients, glioma tissues from glioma patients exhibited a higher expression level of securin (Fig. 1C). These results indicate that the expression of securin is increased in glioma.

Securin overexpression and downregulation in glioma cells. In order to examine the role of securin in gliomas, the present study established securin overexpression and downregulation models in the LCN-229 and U373 cells, respectively. For the overexpression model, securin-expressing pcDNA3.1 plasmids were transfected into the LN-229 cells. Following selection with G418 antibiotics, surviving cells exhibited increased securin expression compared with the empty vector controls ( $\mathrm{P}<0.01$; Fig. 2). Therefore, these LN-229 cells were considered to be stable securin mimic cells. For the securin downregulation model, securin siRNA was transfected into the U373 cells. It was established that the three securin siRNA 
A

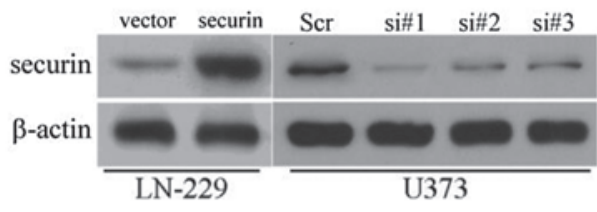

B

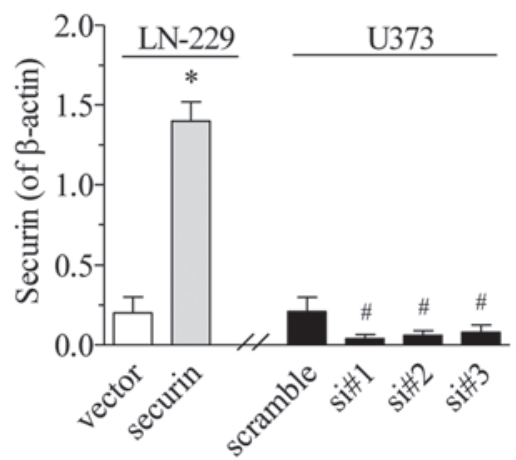

Figure 2. Securin overexpression and downregulation in glioma cells. LCN-229 cells were transfected with either a securin-expressing or empty vector U373 cells were transfected with three different securin-specific siRNAs (si\#1-3) or Scr siRNA. Securin expression in these cells was investigated using western blot analysis $(\mathrm{n}=4)$. (A) Representative immunoblots are shown with (B) the quantified data. ${ }^{\text {"P }}<0.01$ vs. vector group. ${ }^{*} \mathrm{P}<0.05$ vs. Scr group. siRNA, small interfering RNA; Scr, scramble.

A

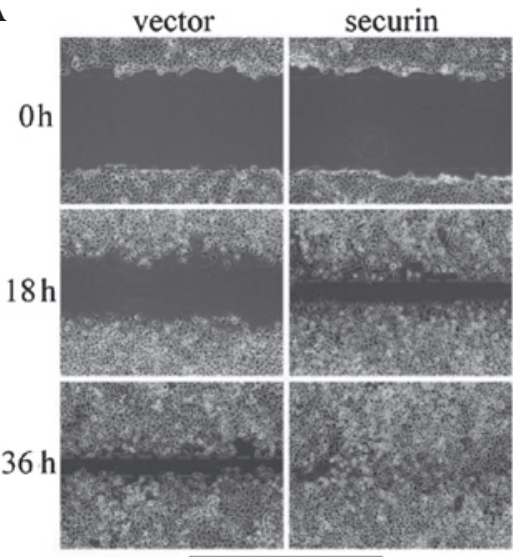

LN-229

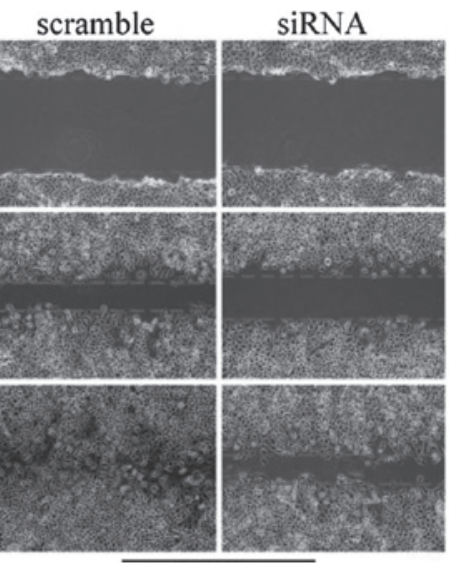

U373
B

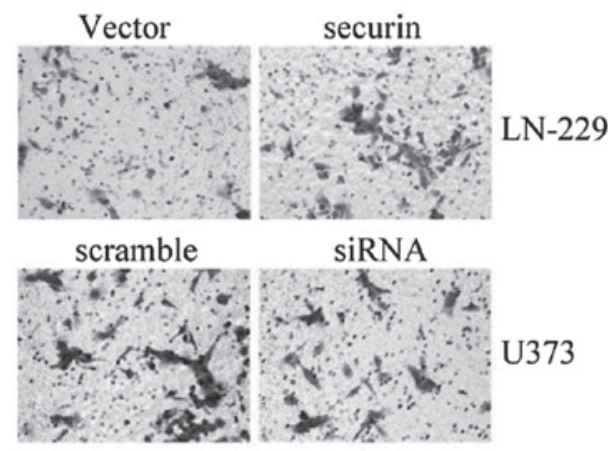

$\mathbf{C}$

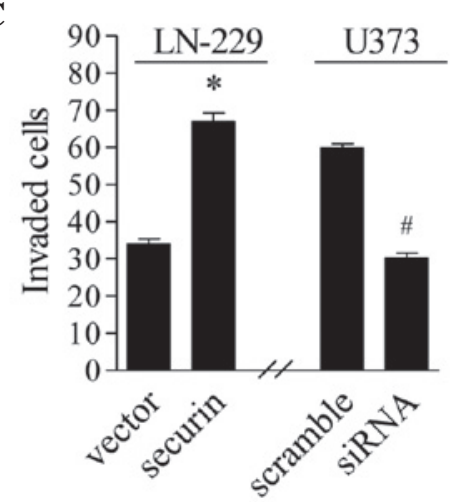

Figure 3. Effect of securin on the migration and invasion of glioma cells. (A) A wound-healing scratch assay (n=6) and (B) a Transwell invasion assay $(n=6)$ were performed in order to evaluate the effect of securin on the migration and invasion of LN-229 and U373 cells, respectively, in which securin was overexpressed or downregulated, respectively. (B) Representative invasion images are shown with (C) the quantified data. "P< $<0.05$ vs. vector group. ${ }^{\#} \mathrm{P}<0.05$ vs. scramble group. siRNA, small interfering RNA.

sequences markedly decreased the level of securin expression $(\mathrm{P}<0.05$; Fig. 2). siRNA sequence \#1 conferred the most marked decrease, and was therefore used in the subsequent experiments.

Securin inhibits migration and invasion in glioma cells. Migration and invasion are important characteristics of tumor metastasis (27). The present study investigated whether securin is involved in glioma metastasis. A classic wound-healing scratch assay was performed in order to establish the role of securin in regulating the migratory ability of the glioma cells. The cell monolayers were scratched to create a wound to monitor the migration ability. The LN-229 cells that overexpressed securin exhibited an increased rate of healing at 18 and $36 \mathrm{~h}$ compared with the empty vector controls. In U373 cells with siRNA sequence \#1-mediated securin-knockdown, 
A

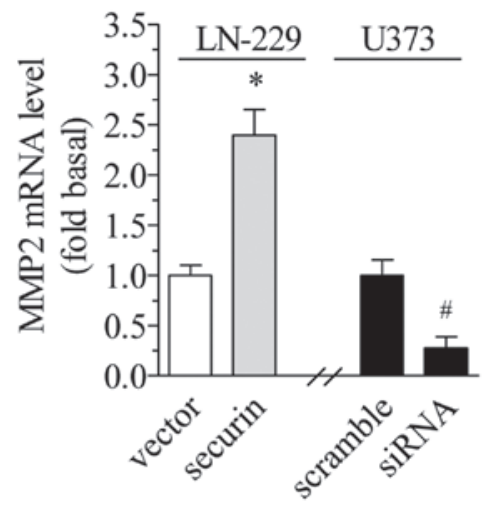

B

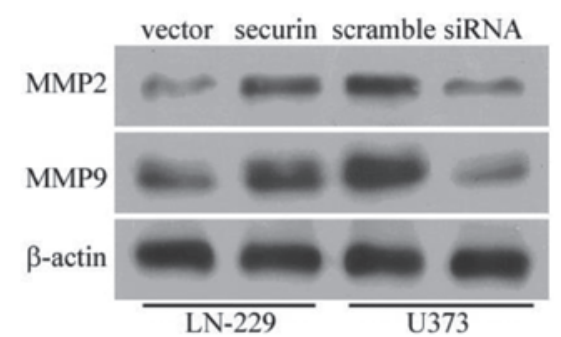

C

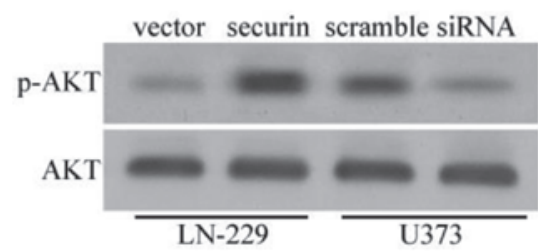

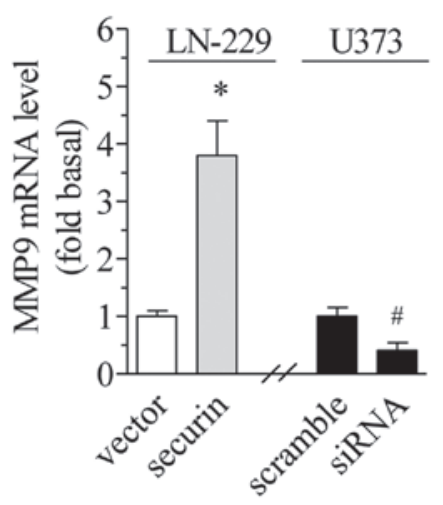
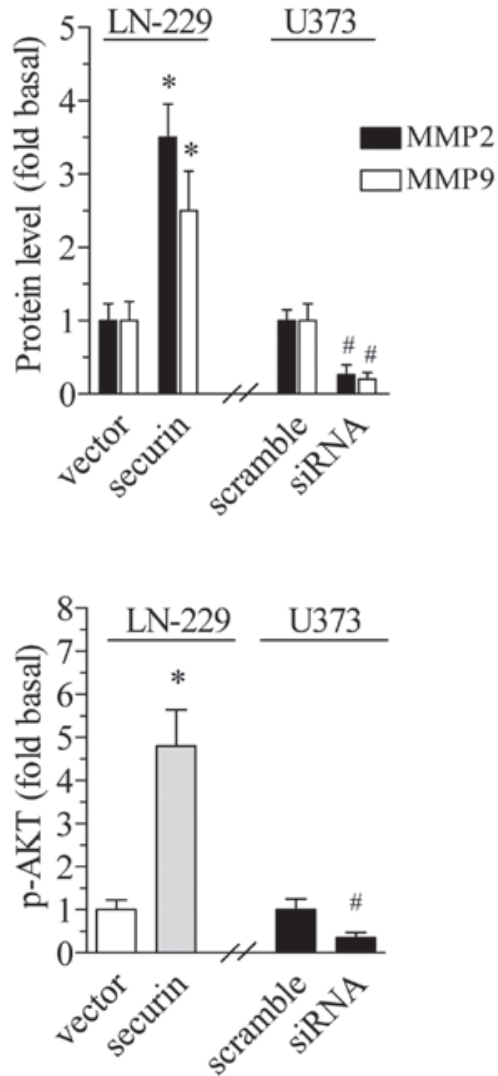

Figure 4. Effect of securin on MMP expression and AKT phosphorylation in glioma cells. Securin was overexpressed or downregulated in LN229 and U373 cells, respectively. The (A) mRNA $(n=6)$ and (B) protein $(n=6)$ expression levels of the MMPs was investigated using quantitative polymerase chain reaction and western blot analysis, respectively. (C) The phosphorylation level of AKT was assessed using western blot analysis (n=6). Representative immunoblots are shown to the left of the quantified data. ${ }^{*} \mathrm{P}<0.01$ vs. vector group. ${ }^{~} \mathrm{P}<0.05$ vs. scramble group. MMP, matrix metalloproteinase; siRNA, small interfering RNA.

healing was markedly inhibited compared with the scramble control (Fig. 3A). These results demonstrate that securin promotes the migration of glioma cells.

The effect of securin on cell invasion was investigated using the Transwell invasion assay. Overall, a higher number of LN-229 cells with securin overexpression traversed the Matrigel-coated polycarbonate membrane compared with the empty vector controls ( $\mathrm{P}<0.05$ : Fig. 3B and C). In U3737 cells, securin-knockdown by siRNA significantly reduced the number of invading cells $(\mathrm{P}<0.05$; Fig. $3 \mathrm{~B}$ and $\mathrm{C})$. Taken together, these results indicate that securin not only promotes the migration ability, but also increases the invasive property of glioma cells.
Securin increases MMPs expression. MMPs degrade ECM components and facilitate tumor invasion and migration (28). The present study investigated whether the level of MMPs changes in response to the expression of securin. The results of the qPCR revealed that securin overexpression in the LCN-229 cells led to a significant increase in the mRNA expression of MMP2 and MMP9 (P<0.01; Fig. 4A). By contrast, securin downregulation in U373 cells significantly decreased the mRNA expression level of MMP2 and MMP9 (Fig. 4A). Similar changes were observed in the protein expression of MMP2 and MMP9. Securin overexpression and downregulation increased and decreased the protein expression of MMP2 and MMP9, respectively $(\mathrm{P}<0.01$ and $\mathrm{P}<0.05$, respectively; Fig. $4 \mathrm{~B}$ ). 
Securin increases phosphorylation of AKT. High AKT activity is associated with tumor growth and invasion (29). Therefore, the present study examined whether securin affected AKT phosphorylation. The results revealed that securin overexpression in LCN-229 cells significantly increased the phosphorylation level of AKT at Ser473 $(\mathrm{P}<0.01)$, while securin downregulation in U373 cells significantly decreased the phosphorylation level of AKT ( $\mathrm{P}<0.05$; Fig. 4C). However, securin overexpression or downregulation did not change the total AKT level.

\section{Discussion}

The present study aimed to evaluate the role of securin in the regulation of migration and invasion in glioma cells, and to identify the potential underlying mechanisms. It was revealed that securin expression was significantly increased in a number of glioma cell lines and tissues. In glioma cells with securin overexpression, the migration and invasion ability was significantly increased. By contrast, in glioma cells with securin downregulation, the migration and invasion ability was significantly decreased. The overexpression and downregulation of securin conferred an increase and decrease in the expression level of MMP2 and MMP9, respectively.

PTTG1/securin is a novel proto-oncogene that serves as a marker of aggressive phenotypes in several types of cancer. In a previous study, a high immunoexpression level of PTTG1 was observed in brain tumor tissues from 88 cases of benign and malignant brain tumors, and was identified to be correlated with malignance (30). In the present study, it was revealed that securin was highly expressed in the glioma tissues obtained from 40 glioma patients compared with in normal brain tissues obtained from brain trauma patients. This finding is consistent with that of a previous study (12) and further increased the sample size in this field. In the four types of glioma cells (LN-229, U87, U251 and U373), securin expression was significantly higher than that in the NHA cells. Therefore, the results demonstrate that securin is highly expressed in glioma.

Migration and invasion are two important steps involved in cancer cell metastasis. The latter ultimately leads to a poor prognosis. Previous studies have reported that an increased expression level of PTTG-1 was correlated with a poor prognosis in patients with glioma (6), esophageal squamous cell cancer (31), medullary thyroid carcinoma (32) and endometrial cancer (33). Therefore, the present study aimed to determine whether securin affects migration and invasion. The results of the Matrigel Transwell invasion assay and wound-healing scratch assay revealed that the overexpression and downregulation of securin increased and decreased glioma cell invasion and migration, respectively. These results indicated a promotive role for securin in the migration and invasion of glioma cells. The promotive effect of securin on migration and invasion has also been observed in other types of cancers. In pituitary macroadenomas, the expression levels of PTTG were significantly higher in invasive pituitary adenomas than in non-invasive pituitary adenomas (34). In a different study, the overexpression of PTTG induced lymph node metastasis in human esophageal squamous cell carcinoma (35). Furthermore, conditioned medium collected from HEK293 cells overexpressing PTTG significantly increased the migration, invasion and tubule formation of human umbilical vein endothelial cells (HUVECs) (25). These previous findings and the data obtained from the present study indicate an important role for securin in glioma migration and invasion.

The invasion of malignant tumors requires the proteolytic degradation of ECM components. Previous data has established that MMPs are major regulators of ECM proteins. In a study by Xia et al (26), the downregulation of PTTG by siRNA markedly decreased the expression of MMP2 and MMP9 in the cutaneous squamous cell carcinoma SCL-1 cell line, and also inhibited cell proliferation. A different study demonstrated that the overexpression of PTTG in metastatic papillary thyroid carcinomas was accompanied by an increased expression level of MMP2 and MMP9 (24). Furthermore, the transient or stable transfection of HEK293 cells with PTTG cDNA has been demonstrated to confer a significant increase in the secretion and expression of MMP2. This secretion in the medium may directly promote the migration and invasion of HUVECs (25). In the present study, the overexpression and downregulation of securin in glioma cells increased and decreased the expression of MMP2 and MMP9, respectively. These results suggest that the increased expression of MMP2 and MMP9 by securin may mediate the migration and invasion of glioma.

The present study also identified that the overexpression and downregulation of securin in glioma cells increased and decreased the phosphorylation level of AKT, respectively. AKT activation has been revealed to be correlated with cell migration and invasion in a number of different tumors. In a previous study concerning glioma, microRNA-149 inhibited cell proliferation and invasion via the blockade of AKT1 signaling (36). In addition, the reduction of AKT phosphorylation by parthenolide has been identified to be accompanied by decreased proliferation, invasion and tumor-induced angiogenesis in glioblastoma cells (37). The results of a further study established that AKT activation also mediated invasion in brain glioblastoma cancer (38). However, data regarding the association between AKT activity and securin is limited. In the human breast cancer MCF-7 cell line, the activation of the PI3K/AKT cascade was reported to mediate the overexpression of PTTG by insulin and insulin growth factor-1 (39). In a study by Pan et al (40), the overexpression of $\gamma$-catenin increased cell mobility and migration, enhanced AKT phosphorylation and led to an increase in the protein level of PTTG. These results indicate a possible interaction between AKT and securin. In the present study, securin exhibited a promotive effect on the phosphorylation of AKT in securin overexpression and downregulation models. These results suggest the existence of a further mechanism in the progression of glioma, which may involve the activation of AKT by securin.

In conclusion, the present study established cell models with securin overexpression or downregulation in order to examine the effect of securin on glioma cell invasion and migration. The results demonstrated an important promotive role for securin in the migration and invasion of glioma cells, which may involve MMPs and AKT phosphorylation.

\section{References}

1. Pei L and Melmed S: Isolation and characterization of a pituitary tumor-transforming gene (PTTG). Mol Endocrinol 11: 433-441, 1997. 
2. Puri R, Tousson A, Chen L and Kakar SS: Molecular cloning of pituitary tumor transforming gene 1 from ovarian tumors and its expression in tumors. Cancer Lett 163: 131-139, 2001.

3. Kakar SS: Molecular cloning, genomic organization and identification of the promoter for the human pituitary tumor transforming gene (PTTG). Gene 240: 317-324, 1999.

4. Zhang X, Horwitz GA, Prezant TR, Valentini A, Nakashima M, Bronstein MD and Melmed S: Structure, expression and function of human pituitary tumor-transforming gene (PTTG). Mol Endocrinol 13: 156-166, 1999.

5. Boelaert K, McCabe CJ, Tannahill LA, Gittoes NJ, Holder RL, Watkinson JC, Bradwell AR, Sheppard MC and Franklyn JA: Pituitary tumor transforming gene and fibroblast growth factor-2 expression: potential prognostic indicators in differentiated thyroid cancer. J Clin Endocrinol Metab 88: 2341-2347, 2003.

6. Genkai N, Homma J, Sano M, Tanaka R and Yamanaka R: Increased expression of pituitary tumor-transforming gene (PTTG)-1 is correlated with poor prognosis in glioma patients. Oncol Rep 15: 1569-1574, 2006

7. Karra H, Repo H, Ahonen I, Löyttyniemi E, Pitkänen R, Lintunen M, Kuopio T, Söderström M and Kronqvist P: Cdc20 and securin overexpression predict short-term breast cancer survival. Br J Cancer 110: 2905-2913, 2014.

8. Demeure MJ, Coan KE, Grant CS, Komorowski RA, Stephan E, Sinari S, Mount D and Bussey KJ: PTTG1 overexpression in adrenocortical cancer is associated with poor survival and represents a potential therapeutic target. Surgery 154: 1405-1416, 2013.

9. Abbud RA, Takumi I, Barker EM, Ren SG, Chen DY, Wawrowsky K and Melmed S: Early multipotential pituitary focal hyperplasia in the alpha-subunit of glycoprotein hormone-driven pituitary tumor-transforming gene transgenic mice. Mol Endocrinol 19: 1383-1391, 2005.

10. Hitomi T, Habu T, Kobayashi H, et al: Downregulation of Securin by the variant RNF213 R4810K (rs112735431, G>A) reduces angiogenic activity of induced pluripotent stem cell-derived vascular endothelial cells from moyamoya patients. Biochem Biophys Res Commun 438: 13-19, 2013

11. Chesnokova V, Kovacs K, Castro AV, Zonis S and Melmed S: Pituitary hypoplasia in Pttg-/- mice is protective for $\mathrm{Rb}+/$ pituitary tumorigenesis. Mol Endocrinol 19: 2371-2379, 2005.

12. Tfelt-Hansen J, Yano S, Bandyopadhyay S, Carroll R, Brown EM and Chattopadhyay N: Expression of pituitary tumor transforming gene (PTTG) and its binding protein in human astrocytes and astrocytoma cells: function and regulation of PTTG in U87 astrocy toma cells. Endocrinology 145: 4222-4231, 2004.

13. Wang L, Wang J, Wang Y, Fu Q, Lei YH, Nie ZY, Qiu J and Bao TY: Protective effect of exogenous matrix metalloproteinase-9 on chronic renal failure. Exp Ther Med 7: 329-334, 2014

14. Toth M, Chvyrkova I, Bernardo MM, Hernandez-Barrantes S and Fridman R: Pro-MMP-9 activation by the MT1-MMP/MMP-2 axis and MMP-3: role of TIMP-2 and plasma membranes. Biochem Biophys Res Commun 308: 386-395, 2003.

15. Gouda HM, Khorshied MM, El Sissy MH, Shaheen IA and Mohsen MM.: Association between matrix metalloproteinase 2 (MMP2) promoter polymorphisms and the susceptibility to non-Hodgkin's lymphoma in Egyptians. Ann Hematol 93: 1313-1318, 2014.

16. Iizuka S, Ishimaru N and Kudo Y: Matrix metalloproteinases: the gene expression signatures of head and neck cancer progression. Cancers (Basel) 6: 396-415, 2014

17. Stetler-Stevenson WG: Type IV collagenases in tumor invasion and metastasis. Cancer Metastasis Rev 9: 289-303, 1990.

18. Lai X, Liao J, Lin W, et al: Inhibitor of DNA-binding protein 1 knockdown arrests the growth of colorectal cancer cells and suppresses hepatic metastasis in vivo. Oncol Rep 32: 79-88, 2014

19. Xia H, Ma YF, Yu CH, Li YJ, Tang J, Li JB, Zhao YN and Liu Y: Aquaporin 3 knockdown suppresses tumor growth and angiogenesis in experimental non-small cell lung cancer. Exp Physiol 99: 974-984, 2014.

20. Chen JS, Li HS, Huang JQ, Zhang LJ, Chen XL, Wang Q, Lei J, Feng JT, Liu Q and Huang XH: Down-regulation of Gli-1 inhibits hepatocellular carcinoma cell migration and invasion. Mol Cell Biochem 393: 283-291, 2014
21. Liu J, Shen L, Yang L, Hu S, Xu L and Wu S: High expression of $\beta 3 \mathrm{GnT} 8$ is associated with the metastatic potential of human glioma. Int J Mol Med 33: 1459-1468, 2014

22. Wen X, Huang A, Liu Z, Liu Y, Hu J, Liu J and Shuai X: Downregulation of ROCK 2 through nanocomplex sensitizes the cytotoxic effect of temozolomide in U251 glioma cells. PLoS One 9: e92050, 2014

23. Liang HS, Zhong YH, Luo ZJ, Huang Y, Lin HD, Luo M, S-Zhan, Su HX, Zhou SB and Xie KQ: Comparative analysis of protein expression in differentiated thyroid tumours: a multicentre study. J Int Med Res 37: 927-938, 2009.

24. Liang H, Zhong Y, Luo Z, Huang Y, Lin H, Luo M, Zhan S, Xie K, Ma Y and Li QQ: Assessment of biomarkers for clinical diagnosis of papillary thyroid carcinoma with distant metastasis. Int J Biol Markers 25: 38-45, 2010.

25. Malik MT and Kakar SS: Regulation of angiogenesis and invasion by human pituitary tumor transforming gene (PTTG) through increased expression and secretion of matrix metalloproteinase-2 (MMP-2). Mol Cancer 5: 61, 2006

26. Xia YH, Li M, Fu DD, Xu SL, Li ZG, Liu D and Tian ZW: Effects of PTTG down-regulation on proliferation and metastasis of the SCL-1 cutaneous squamous cell carcinoma cell line. Asian Pac J Cancer Prev 14: 6245-6248, 2013.

27. Wieczorek E, Jablonska E, Wasowicz W and Reszka E: Matrix metalloproteinases and genetic mouse models in cancer research: a mini-review. Tumour Biol 36: 163-175, 2015.

28. Li H, Yin C, Zhang B, et al: PTTG1 promotes migration and invasion of human non-small cell lung cancer cells and is modulated by miR-186. Carcinogenesis 34: 2145-2155, 2013

29. Li XX, Huang LY, Peng JJ, Liang L, Shi DB, Zheng HT and Cai SJ: Klotho suppresses growth and invasion of colon cancer cells through inhibition of IGF1R-mediated PI3K/AKT pathway. Int J Oncol 45: 611-618, 2014

30. Salehi F, Scheithauer BW, Sharma S, Kovacs K, Lloyd RV, Cusimano MD and Munoz DG: Immunohistochemical expression of PTTG in brain tumors. Anticancer Res 33: 119-122, 2013.

31. Zhang J, Yang Y, Chen L, Zheng D and Ma J: Overexpression of pituitary tumor transforming gene (PTTG) is associated with tumor progression and poor prognosis in patients with esophageal squamous cell carcinoma. Acta Histochem 116: 435-439, 2014.

32. Zatelli MC, Tagliati F, Amodio V, Buratto M, Pelizzo M, Pansini G, Bondanelli M, Ambrosio MR and Degli Uberti EC: Role of pituitary tumour transforming gene 1 in medullary thyroid carcinoma. Anal Cell Pathol (Amst) 33: 207-216, 2010.

33. Kim JW, Song JY, Lee JM, Lee JK, Lee NW, Yeom BW and Lee KW: Expression of pituitary tumor-transforming gene in endometrial cancer as a prognostic marker. Int J Gynecol Cancer 18: 1352-1359, 2008.

34. Jia W, Lu R, Jia G, Ni M and Xu Z: Expression of pituitary tumor transforming gene (PTTG) in human pituitary macroadenomas. Tumour Biol 34: 1559-1567, 2013.

35. Yan S, Zhou C, Lou X, Xiao Z, Zhu H, Wang Q, Wang Y, Lu N, He S, Zhan Q, Liu S and Xu N: PTTG overexpression promotes lymph node metastasis in human esophageal squamous cell carcinoma. Cancer Res 69: 3283-3290, 2009.

36. Pan SJ, Zhan SK, Pei BG, Sun QF, Bian LG and Sun BM: MicroRNA-149 inhibits proliferation and invasion of glioma cells via blockade of AKT1 signaling. Int J Immunopathol Pharmacol 25: 871-881,2012.

37. Nakabayashi $H$ and Shimizu K: Involvement of Akt/NF-кB pathway in antitumor effects of parthenolide on glioblastoma cells in vitro and in vivo. BMC Cancer 12: 453, 2012.

38. Dudley A, Sater M, Le PU, Trinh G, Sadr MS, Bergeron J, Deleavey GF, Bedell B, Damha MJ and Petrecca K: DRR regulates AKT activation to drive brain cancer invasion. Oncogene 33: 4952-4960, 2014

39. Thompson AD III and Kakar SS: Insulin and IGF-1 regulate the expression of the pituitary tumor transforming gene (PTTG) in breast tumor cells. FEBS Lett 579: 3195-3200, 2005

40. Pan H, Gao F, Papageorgis P, Abdolmaleky HM, Faller DV and Thiagalingam S: Aberrant activation of gamma-catenin promotes genomic instability and oncogenic effects during tumor progression. Cancer Biol Ther 6: 1638-1643, 2007. 